\title{
SECURED WIRELESS POWER TRANSMISSION USING RADIO FREQUENCY SIGNAL
}

\author{
B. Renil Randy ${ }^{1}$, M.Hariharan ${ }^{2}$, R. Arasa Kumar ${ }^{3}$ \\ ${ }^{1,2}$ UG Students, ${ }^{3}$ Assistant Professor \\ Velammal College of Engineering and Technology, Madurai
}

\begin{abstract}
:
Anything we transmit without protection is being raid by the intruders. Hence it is necessary to impart security to the signal transmitted. In this paper we present the concept of giving security to the transmitted power signal which is to be received by a receiver in a wireless medium. The present techniques of wireless power transmission consists of a pair of strongly magnetically coupled resonators that allow power transmission of tens of watts over a few meters, but anything without security becomes unreliable. With the goal in achieving security to the power signal, we introduce the concept of encryption decryption algorithm using RF module.
\end{abstract}

\section{INTRODUCTION:}

The inspiration for wireless power comes from wires being cumbersome and messy. With the numerous number of mobile electronics that we use today, there is a huge demand for convenience in supervision of their power supplies. Wireless communication hasmade a massive developmentin the way we interact with communication devices. We also observe that while the techniques of strongly coupled magnetic resonances allowefficient power transfer between a pair of transmitter and receiver coils, the efficiency greatlydeclines upon adding more receivers to the strongly coupled system due to theinteraction between multiple coupled resonators. In tightly coupled resonant wirelesspower transfer, we require a one to one contact between transmitter andreceiver. In addition, in order to improve efficiency of power transfer, we intend to use a lower switching frequency to drive the gate of the MOSFET.

\section{WIRELESS POWER TRANSMISSION (WPT) SYSTEM:}

We cannot imagine the world without electric power. Normally the power is transmitted through wires. This paper depicts an idea to eradicate the hazardous usage of electrical wires which involve lot of confusion in particularly organizing them. Wireless Power Transmission system would completely eliminate the dynamic high-tension power transmission cables, towers and secondary stations between the generating station and consumers and facilitates the interconnection of electrical generation plants on global scale. Mobile transmitters and receivers can be chosen for the Wireless Power Transmission system. The cost for transmission and distribution will be reduced and the cost of electrical energy for the consumers also would be diminished. The power could also be transmitted to the places where the wired transmission is not possible. 


\subsection{VARIOUS SCHEMES OF WIRELESS POWER TRANSFER:}

There are many techniques which are being used to achieve wireless power transfer. Some of the methods are being discussed below.

\subsubsection{WPT using Microwaves:}

In the transmission side, the microwave power source generates microwave power and the output power is managed by electronic restrain circuits. The purpose of the tuner is to match the impedance between the transmitting antenna and the microwave source. The attenuated signals gets divided based on the direction of signal propagation by Directional Coupler. The transmitting antenna emits the power uniformly through free space to the rectenna. In the receiving section, a rectenna receives the transmitted power and translates the microwave power to DC power. The impedance matching circuit and the filter is provided for setting the output impedance of a signal source equal to the rectifying circuit. The rectifying circuit haveSchottky barrier diodes which converts the received microwave power into DC power.

\subsubsection{WPT using Magnetic Resonance:}

In this technique, at first we design an oscillator, which generates the carrier signal to transmit the power. Oscillators are not usually designed to deliver power, thus a power amplifier is added to the oscillator to amplify the oscillating signal. The power amplifier would hand over the output power to the transmission coil. Next, a receiver coil is constructed to receive the transmitted power. However, the power received in the receiver side have an alternating current. Thus, a rectifier is needed to rectify the AC voltage. Finally, an electric load is attached to finish the entire circuit design.

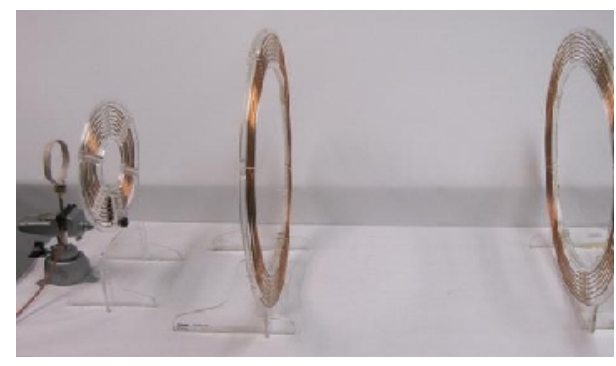

Figure1. WPT using Magnetic Resonance

\subsubsection{WPT using Space Solar power (SSP)Satellites:}

In this method, there are two basic WPT options, one is using radio waves and the other is using light waves, are considered for both long term and short term Space Solar power applications. In the long term, power is transferred to Earth from Geostationary earth orbit (GEO), or further distances in the space. In the near term the power is being transmitted to the moderate distances. By using SSP and WPT technology in near term space science and exploration missions, larger systems are being designed and developed to send power from space to Earth. 


\section{BLOCK DIAGRAM:}

The block diagram for the overall wireless power transceiver is given in the following figure(Fig. 1)

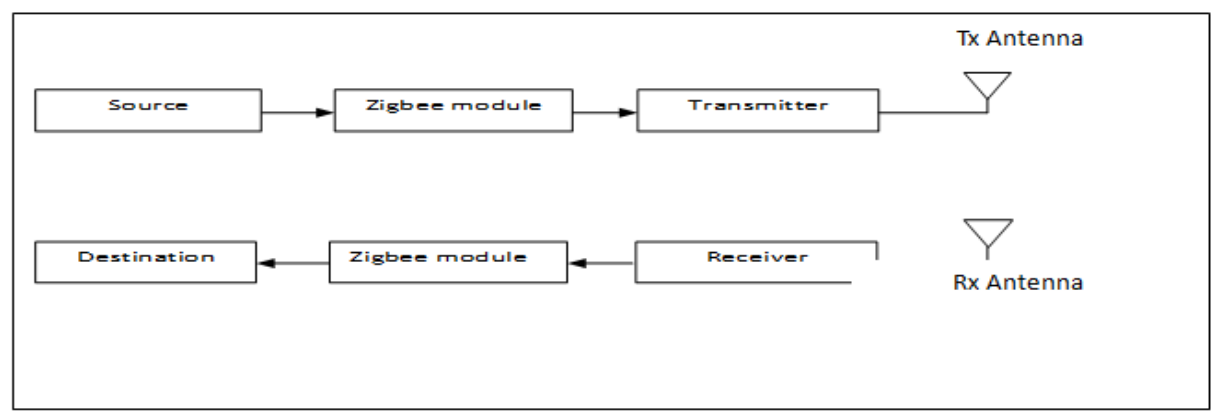

Figure 2. Overall Block Diagram of Wireless power Transceiver

\section{TRANSMITTER:}

The following diagram gives the entire transmitter section of the Wireless Power Transmission. The $230 \mathrm{~V}$ alternating current is fed to the transformer is rectified with bridge rectifier in which the alternating current is converted to Direct Currentand it is filtered and regulated with the voltage regulator. The flow of the signal without any interrupt to the transmitter input is denoted by an LED. It consists of a RESET switch denoted by which is used to reset the programming codes in the picmicrocontroller. The signal is given to the PIC microcontroller PIC16F877A which has 40 pins and it has 5 input output pins and fifteen interrupts and its stability is maintained with a crystal oscillator circuit. This signal will be controlled by the pic microcontroller and the status of the signal is being informed with a help of an LCD display. This signal is being transferred to the RF module through MAX 232. Four MOSFET switches are being connected to each other so that current can pass through, by the switching between the ON and OFF states and the passcode will be generated in MAX 232 and sent through RFtransmitter. 


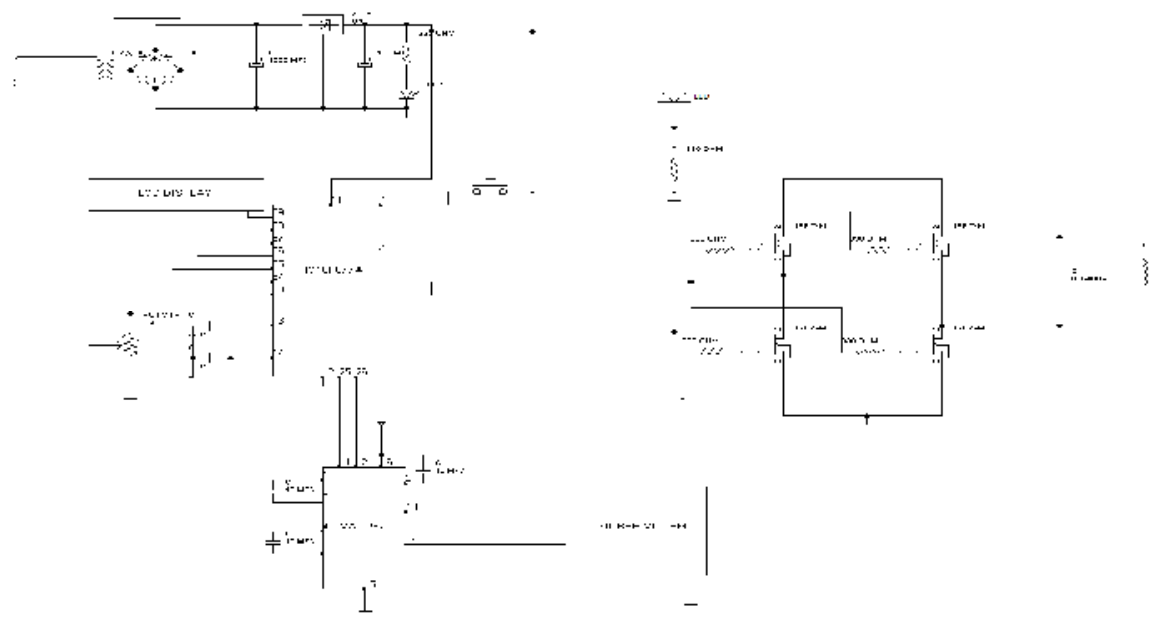

Figure 3. Transmitter diagram

\section{RECEIVER:}

The receiver operation is just the vice versa of the transmitter. The signal is being received to the receiving antenna from the transmitting antenna. The signalthat transmitted from the RF transmitter will reachesthe RF module at the receiver. There is no separate power supply for the receiver section. The current induced in the induction coil is given to the secondary coil of the transformer. In the receiver section, only one MOSFET switch is used which allows current only in the ON state ie, when the signal reaches the RF receiver it will send the passcode to the MAX 232 when the passcode matches then it will trigger the circuit and makes MOSFET switch in ON condition. The current passes to ensure that only the authenticated receiver receives the power signal.

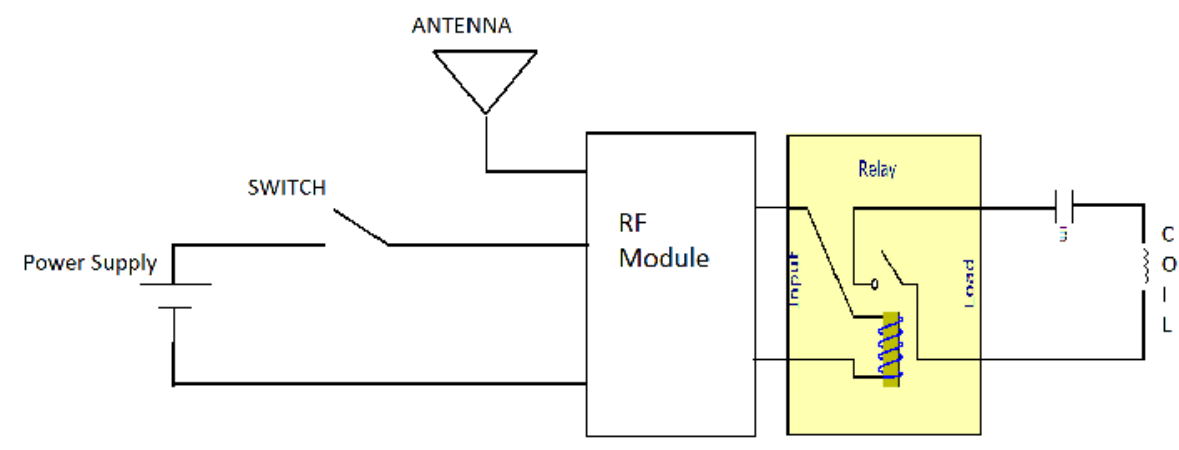

Figure 4. Receiver diagram

\section{RESULTS:}

From the plots given, we come to infer that efficiency of the system has been increased using various parameters when compared to that of the efficiencies of the existing system. Thus the 
concept of presenting the idea of providing security to the transmitted signal in the wireless medium and receiving is attained is done for a limited space in small scale. This would also become base for the further research in transmitting and receiving signals in a large scale through anyone one of the above listed techniques.

Table 1. Result of current by varying distance of receiver

\begin{tabular}{|c|c|c|}
\hline $\begin{array}{c}\text { Distance } \\
\text { (feet) }\end{array}$ & $\begin{array}{c}\text { Voltage } \\
\text { (Volts) }\end{array}$ & $\begin{array}{c}\text { Current } \\
(\mathbf{m A})\end{array}$ \\
\hline 3 & 4 & 500 \\
\hline 2 & 4.5 & 550 \\
\hline 1 & 5 & 600 \\
\hline 0.5 & 5.5 & 750 \\
\hline $\begin{array}{c}\text { Maximum } \\
\text { closest } \\
\text { position }\end{array}$ & 6 & 1000 \\
\hline
\end{tabular}

From the above observations, the power transferred from the transmitter to the receiver is maximum at the closest position and when the distance between them is increased, the power received in the receiver gets reduced.

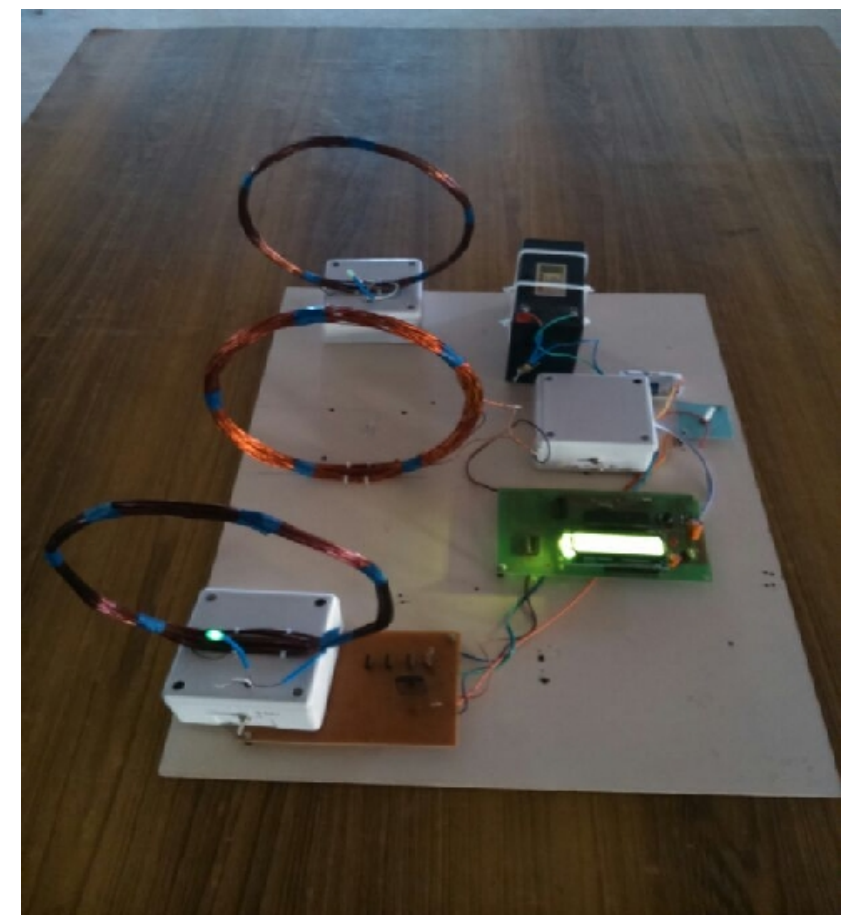

When the transmitter is switched to the port of the receiver A, we are able to observe that the LED on the receiver A glows and the LED in the receiver coil B doesn't glow since receive B is not the authenticated user to use the power signal. Any metal sheet obstacle when placed between 
the transmitter and the receiver, it is found that the power is transmitted to the authenticated user as the metal sheet is a conductor.

Table 2. Result of temperature with varying distance

\begin{tabular}{|c|c|}
\hline Distance (feet) & Temperature $\left({ }^{\circ} \mathbf{C}\right)$ \\
\hline 3 & 27 \\
\hline 2 & 27.7 \\
\hline 1 & 28.2 \\
\hline 0.5 & 29.5 \\
\hline Maximum closest position & 30 \\
\hline
\end{tabular}

From the above tabulation, we find that the temperature in the receiver coil gets increased when the when the distance the distance between the transmitter and the receiver is decreased

Table 3. Result on transmitter coil heating

\begin{tabular}{|c|c|}
\hline PWM On duty Time & $\begin{array}{c}\text { Transmitter coil heating } \\
\left({ }^{\circ} \mathbf{C}\right)\end{array}$ \\
\hline $100 \%$ & 45 \\
\hline $90 \%$ & 43 \\
\hline $80 \%$ & 41 \\
\hline $70 \%$ & 40 \\
\hline $60 \%$ & 38 \\
\hline
\end{tabular}

From the above observations, we find that at the temperature of the transmitter coil is high when the ON duty time of the Pulse width modulation (PWM) is maximum. 


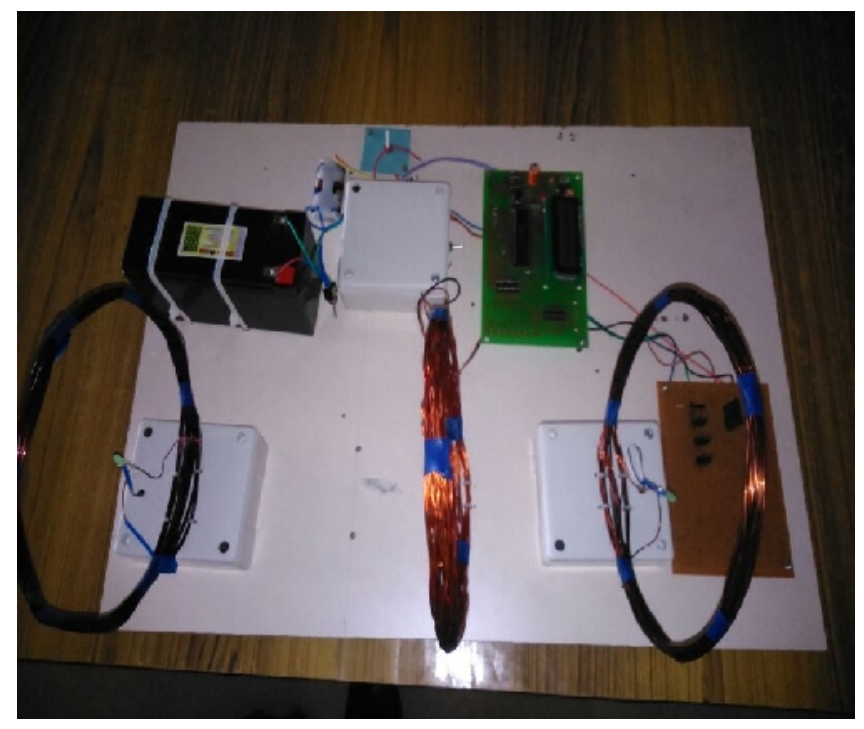

When the transmitter is neither switched to the receiver of port A nor port B ie, the switch is held in the middle. Then both the receiver A and B can't receive the power since both the receivers are not authenticated even though the power is transmitted in the air and the coil induces it. The four MOSFET which is being connected in series helps in switching the current. The amount of power received in the receiver varies when the distance between the transmitter and the receiver varies as discussed.

\section{CONCLUSION:}

We have placed two receivers with decoders which decodes the passcode given as coding to the RF module in the transmitter section. The receiver which has the matched passcode decoded has received the power signal successfully. The receiver with the decoder decoding a passcode mismatch failed to receive the power signal. Our project can also be implemented in Online Electric Vehicle (OLEV). OLEV wireless charging system consists of a road-side Power Inverter to bring electricity from the grid to the road embedded power tracks. A proprietary Roadway Infrastructure consisting of Road-embedded Power tracks installed at selected locations of the route.A proprietary Pick-Up and Regulator kit for the wireless power transfer installed in or under the electric vehicle. Hence in this system the unauthorised users are denied permission to use the power source using the key idea of the project.

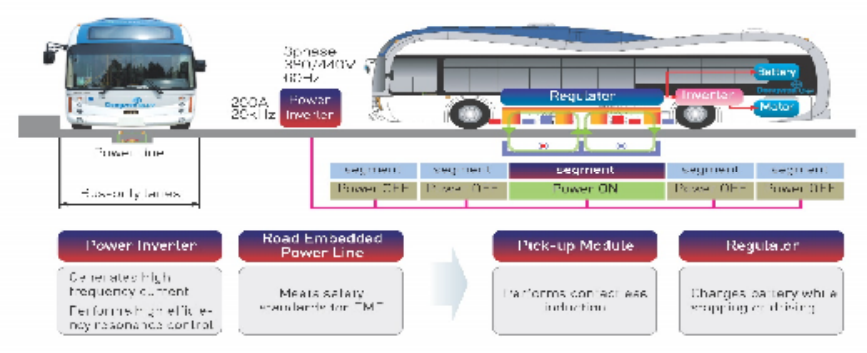




\section{REFERENCES:}

[1] S. Kopparthi, Pratul K. Ajmera, "Power delivery for remotely located Microsystems,"

[2] Proc. of IEEE Region 5, 2004 Annual Tech. Conference, 2004 April 2, pp. 31-39.

[3] Nikola Tesla, "The Transmission of Electrical Energy Without Wires as a Means for Furthering Peace," Electrical World and Engineer. Jan. 7, p. 21, 1985.

[4] T. Deyle and M. Reynolds, "Surface based wireless power transmission and bidirectional communication for autonomous robot swarms" in Proceedings IEEE Conference on Robotics, 2008.

[5] M. Ghovanloo, et al., "A Wide-band Power-efficient Inductive Wireless Link for Implantable Microelectronic Devices Using Multiple Carriers,” IEEE Trans. Circuits and Systems, vol. 54, no. 10, October 2007.

[6] K. M. Silay, et al., "Improvement of Power Efficiency of Inductive Links for Implantable Devices," Research in Microelectronics and Electronics, pp. 229-232, June 2008.

\section{AUTHORS:}

B. Renil Randy is doing his B.E. degree in Velammal College of Engineering and Technology, Madurai, India. He is interested in the area of Wireless Communication and Signal Processing.

M. Hariharanis doing his B.E. degree in Velammal College of Engineering and Technology, Madurai, India. He is intere sted in the area of Wireless Communication and specialized in Wireless Power Transmission.

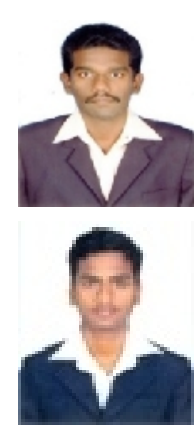

R. Arasa Kumar,M.E.is working as Assistant Professor in Velammal College of Engineering and Technology, Madurai. He also has teachin g experience at Kalasalingam Institute of Technology, Krishnakoil. He was graduated at MepcoSchlenk Engineering College, Sivakasi.

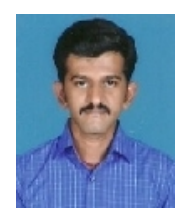

